\title{
OORT'S COMETARY CLOUD IN THE LIGHT OF MODERN COSMOGONY
}

\author{
V.S. SAFRONOV
}

\begin{abstract}
Although the existence of Oort's cometary cloud has been generally accepted, his hypothesis on its origin has been repeatedly called into question, in particvlar because of the large mass that Jupiter would also have simultaneously ejected out of the solar system. However, the extremely slow growth of particles in regions of small density seems to rule out that comets condensed "in situ" at their present large distances. Also, the accumulation of interstellar grains in satelite disks orbiting around the primitive solar nebula seems an "ad hoc" hypothesis that cannot be proved or disproved. Therefore, the most reasonable hypothesis is that comets were ejected from the region of the giant planets as a natural by-product of their accretion.
\end{abstract}

For a quarter of a century, Oort's $(1950,1951)$ concept of a cometary cloud has been used by many specialists as a reasonable working model. Oort supposed that, during its formation, Jupiter caused many bodies to be ejected From the asteroidal belt by its gravitational perturbations. A small fraction of these hodies remained at the outskirts of the solar system and formed the cometary cloud. It was subsequent $1 y$ shown that all the giant planets ejected bodies into this cloud. Recently, however, the efficiency of Oort's mechanism has been called into question. It was pointed out that the mass of comets thrown completely out of the solar system would be much greater than that ejected into the cometary cloud (Ostriker 1972). Furthermore, conservation laws dictate that the escape of some mass from the system leads to the contraction of remainder, i.e., the planets should move closer in to the sun (Cameron 1973). These doubts have caused other hypotheses on cometary formation to be revived, with the comets forming at their present large distances from the sun. It is therefore useful to analyze which of these hypotheses is to be preferred.

1. Comets are bodies ejected from the region of the giant planets. Our study of the accumulation of giant planets (Safronov 1972a) has shown that as the planets grew they caused the velocities of bodies in their vicinity to be increased. At the end of the process the mass of bodies that had acquired escape velocity during encounters with a planet exceeded by several times the mass that had fallen on to the planet. It was also evaluated (Safronov 1972b) that Uranus and Neptune could have grown to their present sizes only if the 
initial mass of solid material in their vicinity was about an order of magnitude greater than the present masses of these planets. The ejection of bodies from the solar system was therefore a natural by-product of the accumulation process of the giant planets. A small fraction (about 10-2) of these bodies had their orbits changed due to stellar perturbations and formed the cometary cloud. It must be emphasized that the bulk of the ejection is due to Jupiter, although a significant part is due to Saturn. The ejection of mass, amounting to at most $300 M_{\oplus}$ (about $2 M_{\oplus}$ of it going into the cometary cloud) would not lead to unacceptable shrinking of the planetary orbits. The orbits of Neptune and Uranus would not change very significantly because these planets ejected bodies both outwards and inwards, many of the latter then being thrown out of the system by Jupiter and Saturn.

2. Comets condensed in situ at their present large distances. This hypothesis is often mentioned (e.g., Whipple 1976) but has never been developed in detail. Its main difficulty is the extremely slow growth of particles in regions of very low density. The untenability of the hypothesis was pointed out by Öpik (1973), for if one solar mass is uniformly distributed over a sphere of radius $50,000 \mathrm{AU}$, the mean density is $10^{-21} \mathrm{~g} \mathrm{~cm}^{-3}$, of which 2 percent would be condensable material. During $5 \times 10^{9}$ years particles could grow only to a radius $\sim 10^{-6} \mathrm{~V} \mathrm{~cm}$, where the velocity $\mathrm{V}$ is given in $\mathrm{cm} \mathrm{s}^{-1}$.

3. Comets were formed in satellite disks orbiting around the primitive solar nebula. This hypothesis was suggested by Cameron $(1973 \mathrm{a}, 1973 \mathrm{~b})$ in a purely qualitative form. It is assumed that not all of the collapsing interstellar cloud became a part of the primitive solar nebula. Because of turbulent motions some sections had angular momentum large enough to form separate gaseous condensations orbiting the solar nebula. In these cold satellite nebulae comets would be formed by the accumulation of interstellar grains. So little is known at present about the evolution of the solar nebula that we can neither prove no disprove such a hypothesis. Calculations by Larson (1972), confirmed later by Black (1976), have shown that collapse of a rotating nebula would lead to the formation, not of a single central starlike condensation, but of an unstahle ring that would divide into two or more protostars. The whole picture becomes very complex. Furthermore, the hypothesis is not supported by facts, for observations do not reveal the secondary disks of comets. From a methodological point of view hypotheses of this type can be justified only when there are no others with a more solid basis.

We conclude that at the present time the most reasonable idea is that the cometary cloud formed as a result of the gravitational ejection of bodies from the vicinity of the giant planets.

\section{REFERENCES}

Black, D. 1976, preprint .

Cameron, A.G.W. 1973a, Center for Astrophys. Prepr. No. 21.

Cameron, A.G.W. 1973b, Icarus, 18, 407.

Larson, R. B. 1972, in On the Origin of the Solar System, ed. H. Reeves, CNRS, Paris, p. 142 .

Oort, J. H. 1950, Bull. Astron. Inst. Neth. 11, 91.

Oort, J. H. 1951, observatory, 71, 129.

Ostriker, J. P. 1972, in On the Origin of the Solar system, ed. H, Reeves, CNRS, Paris, p. 163.

Öpik, E. J. 1973, Astrophys. Space Sci., 21, 307.

Safronov, V. S. 1972a, IAU Symp. No. 45, p. 329.

Safronov, V..S. 1972b, Evolution of the Protoplanetary cloud and Formation of the Earth and the Planets (translated from the Russian). Nat. Tech. Inf. Service, Springfield, Virginia.

Whipple, F. L. 1976, in Cosmochemistry of the Moon and planets, ed. A. P. Vinogradov. 